\title{
AVALIAÇÃO DA COLOCAÇÃO DE PROTETORES AURICULARES EM GRUPOS COM E SEM TREINAMENTO
}

\author{
Evaluation of hearing-protector insertion in groups \\ with and without training
}

\author{
Cláudia Giglio de Oliveira Gonçalves (1), Christiane Marques do Couto (2), \\ Juliana Malteze Carraro ${ }^{(3)}$, Bianca Santos Leonelli ${ }^{(4)}$
}

\begin{abstract}
RESUMO
Objetivo: analisar a eficácia da colocação de diferentes protetores auriculares em sujeitos treinados e não-treinados e verificar a importância e a necessidade da realização de um treinamento para a colocação adequada do Equipamento de Proteção Individual auricular. Métodos: investigou-se a atenuação de quatro modelos diferentes de protetores auriculares (inserção de espuma, inserção de silicone três flanges, inserção oliva e tipo concha) por meio de duas estratégias: audiometria em campo livre e medidas com microfone sonda, com e sem protetor auricular para comparação e determinação da vedação de ruído. Avaliou-se dois grupos, Grupo 1, sujeitos que receberam treinamento e orientação em relação ao uso e colocação do EPI auricular (23) e o Grupo 2, sujeitos não-treinados (8) universitários que nunca utilizaram o EPI auricular. Resultados: no grupo 1, as diferenças dos limiares auditivos sem e com protetor auricular avaliadas pelos dois métodos apresentaram, em geral, valores de atenuação superiores ao do grupo 2. O protetor auricular concha apresentou valores médios de atenuação superiores aos demais tipos; e o protetor auricular oliva apresentou valores menores. Conclusão: o treinamento quanto ao uso dos protetores auriculares é necessário e deve ser incluído nos Programa de Conservação Auditiva (PCA).
\end{abstract}

DESCRITORES: Dispositivos de Proteção das Orelhas; Perda Auditiva Provocada por Ruído; Saúde do Trabalhador; Audição

\section{INTRODUÇÃO}

Encontra-se na legislação trabalhista brasileira (Consolidação das Leis do Trabalho) a orientação quanto à utilização de equipamentos de proteção individual (EPI) quando forem identificados nos ambientes de trabalho riscos potenciais de dano

(1) Fonoaudióloga; Professora do Programa de Mestrado em Distúrbios da Comunicação da Universidade Tuiuti do Paraná, UTP, Curitiba, PR; Especialista em Audiologia; Doutora em Saúde Coletiva.

(2) Fonoaudióloga; Professora do curso de Fonoaudiologia da Universidade Estadual de Campinas, UNICAMP, Campinas, SP; Especialista em Audiologia; Doutora em Ciências pela Faculdade de Medicina da Universidade de São Paulo.

(3) Fonoaudióloga; Intermedici; Especialista em Audiologia.

(4) Fonoaudióloga; Brumed, Itapeva, SP; Especialista em Audiologia.

Conflito de Interesses: INEXISTENTE à saúde. Porém, a utilização de EPI deverá ser realizada quando houver inviabilidade de adoção de medidas de proteção coletiva ou até que estas sejam efetivamente implantadas (NR - Norma Regulamentadora n. 9 -9.3.5.4) ${ }^{1}$.

$\mathrm{Na}$ utilização do EPI, deve-se considerar, segundo a NR 9, as normas legais e administrativas (item 9.3.5.5) que envolvem a seleção adequada, conforto, uso, higienização, manutenção, orientação e avaliação dessas medidas adotadas sobre os EPI, garantindo-se as condições de proteção originalmente estabelecidas pelos fabricantes.

No entanto, o que se observa em grande parte das indústrias que adotam os equipamentos de proteção individual é a dificuldade na avaliação de sua correta utilização pelos trabalhadores ${ }^{2}$. Isso, sobretudo, em relação aos protetores auriculares, recomendados quando os níveis de pressão sonora excederem 85 dBA por 8 horas nos ambientes de trabalho. Essa dificuldade acontece pela variedade 
de tipos de protetores auriculares e pela dificuldade de manuseio dos mesmos sem um treinamento adequado ${ }^{3}$. Há uma diferença no valor da atenuação do ruído entre os protetores auriculares e essa diferença pode aumentar dependendo da colocação do protetor ${ }^{3}$.

Somente a distribuição dos protetores auriculares e a obrigatoriedade do uso, não são suficientes para a preservação da audição ${ }^{2}$. Programas que envolvam os trabalhadores devem ser implementados. Os Programas de Conservação Auditiva visam a garantia da preservação da audição de trabalhadores, através de ações envolvendo a análise do ambiente de trabalho e o controle dos agentes otoagressivos, o estudo do perfil auditivo e a implementação de ações educativas, não se restringindo unicamente a realização de audiometrias e distribuição de protetores auriculares ${ }^{2,4}$.

Mesmo sendo obrigatório aos fabricantes de EPI realizarem a avaliação dos mesmos ${ }^{5}$, comprovando, assim, sua eficiência, isso não é garantia de que o trabalhador irá beneficiar-se de sua proteção, pois a colocação inadequada comprometerá os objetivos do EPI, e no caso dos protetores auriculares, que já apresentam diferenças quanto à proteção, dependendo do tipo utilizado, se mal colocados, sua eficácia é comprometida ${ }^{3,6}$.

Independente do tipo e modelo (existem mais de 1000 ), os protetores auriculares devem ser escolhidos de acordo com a atenuação de ruído necessária, o conforto ao usuário, ambiente de trabalho e custo. Porém, o melhor protetor auricular será aquele que o trabalhador melhor se adaptar ${ }^{6}$.

A colocação inadequada de protetores auriculares faz com que o ruído consiga atingir a orelha em níveis superiores aos estabelecidos com o uso do protetor auricular ${ }^{3,6}$.

Estudo nos Estados Unidos sobre causas das diferenças individuais da atenuação do ruído pelos protetores auriculares, explorando as barreiras culturais e de linguagem que estão por trás do uso adequado dos protetores auriculares, concluiu que os trabalhadores com dificuldades de compreensão da linguagem obtiveram menor desempenho com o uso do protetor auricular ${ }^{7}$.

Para a determinação da redução do ruído dos protetores auriculares é necessário realizar ensaios de atenuação de ruído, seguindo-se normas nacionais ou internacionais, por métodos objetivos ou subjetivos $^{8}$.

Para avaliação da atenuação real obtida no indivíduo têm sido propostos métodos objetivos e subjetivos. O método subjetivo melhor aceito para avaliação da atenuação de protetores auriculares é o Real-Ear Attenuation at Threshold - REAT, no qual são apresentados diversos estímulos de ruído rosa a um sujeito numa cabina acústica sem e com protetor auricular que indicará quando escutou o sinal acústico, para comparar-se os limiares auditivos. O questionamento que se faz a esse procedimento (REAT) é quanto a sua aplicabilidade numa situação real de trabalho. Além disso, a audiometria em campo livre possui grande variabilidade em seus resultados possuindo um desvio-padrão de 4 a 6 $\mathrm{dB}^{9,10}$.

Alguns estudos vêm utilizando o método objetivo MIRE (Microphone-in-Real-Ear), que utiliza um microfone sonda colocado próximo à membrana timpânica; faz-se a mensuração do sinal acústico (ruído rosa) apresentado em intensidade de $85 \mathrm{~dB}$ numa distância de $80 \mathrm{~cm}$ da pessoa testada. São registradas as diferenças entre os valores com e sem protetores auriculares. Ao contrário das medidas em campo livre, esse procedimento possui uma variabilidade pequena ${ }^{10}$.

Muitas pesquisas vêm sendo desenvolvidas nos últimos anos para avaliar a atenuação dos protetores auriculares. Alguns trabalhos ${ }^{10-13}$ compararam as avaliações de atenuação utilizando o procedimento de microfone sonda e a REAT. A atenuação encontrada pelo método de microfone sonda foi semelhante aos dados do fabricante, com diferença em média de $2 \mathrm{~dB}$ a menos na atenuação pelo método REAT. Autores sugeriram que para maior confiabilidade de respostas, utilize-se mais de uma maneira para avaliar os protetores auriculares e que se considerem as questões individuais dos trabalhadores ${ }^{14,15}$.

Avaliando os dados encontrados na literatura, o objetivo desse estudo foi analisar a importância e a necessidade de treinamentos para uma eficiente colocação de protetores auriculares, utilizando-se procedimentos objetivos de avaliação e verificar a importância e a necessidade da realização de um treinamento para a colocação adequada do EPI auricular.

\section{MÉTODOS}

Trata-se de um estudo transversal, com uma amostra composta de dois grupos avaliados na Clínica-Escola da Universidade Metodista de Piracicaba e que assinaram o Termo de Consentimento Livre e Esclarecido. O grupo 1 foi composto por 23 trabalhadores todos do sexo masculino expostos a ruído ocupacional (indústria metalúrgica) e que receberam orientações e treinamento sobre a utilização de protetores auriculares ministrada por técnico de segurança e fonoaudióloga. A média de idade desse grupo é de 37 anos, o grau de escolaridade é de 8 anos e a média de tempo de serviço de 17,6 anos. O grupo 2 foi composto por oito 
estudantes universitários não trabalhadores da indústria (dois do sexo masculino e seis do sexo feminino), que nunca passaram por treinamento sobre o uso do EPI auricular, com média de idade de 21 anos.

Os protetores auriculares testados foram: tipo concha (Marca 3M), inserção moldável de espuma descartável (marca $3 \mathrm{M}$ ), inserção de silicone com três flanges (marca Pomp) e inserção de silicone modelo oliva (marca Quiet).

Foram utilizados dois métodos convencionalmente utilizados para avaliação de próteses auditivas e adaptados para avaliação do ruído que é atenuado pelos protetores auriculares: audiometria em campo livre com e sem protetor e medidas de microfone-sonda, descritos na literatura quando aplicado às medidas de atenuação como REAT (Real-Ear Attenuation at Threshold) e MIRE (Microphone-in-Real-Ear) ${ }^{9}$.

Não foi exigido repouso auditivo, pois, como as medidas são relativas, estas não seriam influenciadas por mudança temporária do limiar.

Foi escolhida para a avaliação somente a orelha direita. Tal escolha foi devida ao local do teste, que apresentava maior possibilidade de reverberação do lado esquerdo. A priori foi realizada a meatoscopia nessa orelha para observar a existência de obstrução por cera ou corpo estranho no meato acústico externo, sendo esse um critério de exclusão.

Em seguida, partiu-se para a mensuração com microfone sonda utilizando um equipamento computadorizado Hearing Aid Analyzer MS 40 (Interacoustics). Trata-se de um conjunto dotado de uma unidade central, composta por computador, um sistema de microfones (um de referência e um microfone-sonda com um diâmetro interno de 1,8 mm), um compressor de alta resolução ligado a um altofalante, um monitor de vídeo e uma impressora para registro dos resultados. O equipamento produz, por meio do alto-falante, um sinal em campo livre e um microfone de referência que assegura que o nível de pressão sonora, inicialmente ajustado, permaneça inalterado durante toda a mensuração. Ele possibilita a realização do exame na faixa de frequência de 125 a $8000 \mathrm{~Hz}$, com uma opção de resolução de frequência de 12 ou 24 passos/oitava. A precisão das frequências medidas é de $\pm 3 \%$. Para a realização do teste, o equipamento deverá gerar como estímulo tom modulado em frequência ("tom warble") em torno de $3 \%$. O equipamento possui uma faixa de intensidade de teste de 50-90dB NPS com acurácia de $\pm 1,5 \mathrm{~dB}$, com uma distorção harmônica do estímulo menor do que $1 \%$. A faixa de intensidades medidas com o microfone-sonda é de 40-140dBNPS, com incerteza de $\pm 2 \mathrm{~dB}$.
Esse equipamento normalmente é utilizado para avaliação de próteses auditivas e permite o registro do Nível de Pressão Sonora (NPS) ao nível da membrana timpânica, sendo realizadas as medidas REUR (Real Ear Unaided Response traduzida como Resposta de Ressonância da Orelha Externa), o REOR (Real Ear Occluded Response traduzida como Resposta de Oclusão na Orelha Externa) expressas em gráficos compostos no eixo vertical de valores de dBNPS e no eixo horizontal de valores em $\mathrm{kHz}$.

Para iniciar a mensuração com microfone sonda é necessário realizar a calibração da unidade microfônica. Após a calibração, o sujeito foi colocado a uma distância de $40 \mathrm{~cm}$ do alto falante, num ângulo de $0 \% 0^{\circ}$ azimute e orientado para não movimentar a cabeça durante todo o procedimento do teste ${ }^{16}$. O microfone sonda foi colocado no meato e obtido o REUR, que é a resposta de ressonância da oreIha externa em função da frequência obtida em um ponto específico do meato acústico externo para um determinado campo sonoro, com o meato acústico não ocluído. O nível de pressão sonora de entrada foi de $80 \mathrm{dBNPS}$ com o tom puro modulado em frequências (tom warble) fazendo uma varredura de frequências de 125 a $8000 \mathrm{~Hz}$ e analisadas as frequências de 500, 1000, 2000 e $4000 \mathrm{~Hz}$.

Depois de registrada a curva de ressonância, foi solicitado, sem instrução específica por parte do avaliador, para que o sujeito colocasse seu protetor auricular na orelha que estava com o microfone sonda, com cuidado para não deslocá-lo. Realizouse, então, outro registro com mesmo estímulo para se observar a curva com a utilização do mesmo nível de entrada, REOR que é a reposta da orelha ocluída. Foram analisadas novamente as respostas nas frequências de 500, 1000, 2000 e $4000 \mathrm{~Hz}$.

Foi calculada a diferença entre o REUR e REOR (denominada aqui REIL- Real-Ear Insertion Loss), que é a diferença em decibel entre a medida com e sem o protetor auricular inserido no MAE do sujeito.

Logo após, foi retirado o microfone sonda e mantido o protetor auricular na mesma posição para a realização da audiometria em campo livre. Essa medida foi realizada na mesma sala acusticamente tratada, onde foi realizada a medida com microfonesonda, utilizando-se um audiômetro AD29 (Kamplex) com saída para campo livre e fone TDH 49.

Para realizar essa medida, o sujeito foi colocado a um metro da caixa de som e posicionado a $0^{\circ}$ azimute. Foram pesquisados os limiares auditivos da orelha direita com e sem protetor auricular, nas frequências de 500, 1000, 2000 e $4000 \mathrm{~Hz}$. O estímulo utilizado foi o tom puro modulado em frequências (tom warble). Foi utilizado o método psicométrico 
dos limites (técnica descendente-ascendente) com variação de $5 \mathrm{~dB}^{17}$.

Foi utilizado o mascaramento com ruído narrow band na orelha não testada (orelha esquerda), sendo que, naqueles sujeitos que tinham audição normal foi utilizado 40dBNA e, no caso dos sujeitos com perda auditiva, foi utilizado o nível de ruído maior ou igual a 50dBNA. Foi calculado o REAT (REAL-Ear Attenuation at Threshold), que é a atenuação real obtida pela diferença entre os limiares com e sem protetor auricular na audiometria em campo livre.

$\mathrm{Na}$ pesquisa da mensuração com microfone sonda a atenuação foi obtida através da diferença entre os valores de pressão sonora sem e com o protetor auricular (REIL). Na audiometria em campo livre a atenuação foi obtida pela diferença dos limiares auditivos com e sem o uso do protetor auricular.

O projeto foi aceito pelo Comitê de Ética da Universidade Metodista de Piracicaba sob o número 20/04.

Para a análise dos dados, as diferenças nos limiares auditivos dos dois grupos de sujeitos sem e com os protetores auriculares foram expressas em médias. Analisou-se a similaridade entre as médias para constatar se ocorreu atenuação do ruído e como se comportaram cada grupo quanto à colocação do protetor auricular que apresentasse melhor atenuação, por meio do teste Wilcoxon (nível de significância de 5\%), adequada para a amostra estudada, controlando as diferenças no número de sujeitos por grupos.

Os achados também foram analisados em função do tipo de protetor auricular utilizado comparando-se o desempenho de cada modelo em função de cada método de avaliação utilizado.

\section{RESULTADOS}

Analisou-se a diferença dos limiares auditivos sem e com protetores auriculares após sua colocação pelo usuário, pelos dois métodos, em campo livre (REAT) e por microfone-sonda (MIRE) com o cálculo do REIL e comparou-se os resultados nos dois grupos (grupo 1: trabalhadores que utilizam protetores auriculares e grupo 2: estudantes sem experiência no uso de protetores auriculares).

Foi realizada a média dos REAT e REIL para as frequências 500, 1000, 2000 e 4000Hz (Tabela 1).

No grupo 1, o REAT e o REIL, apresentaram, em geral, valores superiores ao grupo 2 .

Quando se analisa o REAT e o REIL para o grupo 1 foi encontrado, na frequência de 4000 $\mathrm{Hz}$, valores com uma diferença significante entre os métodos $(p=0,002)$, nível de significância de $5 \%$, teste Wilcoxon). No grupo 2 , houve diferença significante para as frequências de 500 e $1000 \mathrm{~Hz}$ (ambas com $\mathrm{p}=0,049951$ ).

Investigou-se cada tipo de protetor auricular, pelos dois métodos, nos dois grupos (Tabela 2).

Em relação ao protetor auricular do tipo concha, considerado de fácil colocação, observou-se, em geral, maior valor do REAT e REIL, demonstrando maior atenuação.

Em relação ao protetor auricular do tipo espuma descartável, observou-se um maior REAT e REIL para o grupo 1. Os valores da REIL nas frequências de 500 a $2000 \mathrm{~Hz}$ foram nulos para o grupo 1 . Em relação ao protetor auricular do tipo silicone de três flanges, as frequências 500 e $2000 \mathrm{~Hz}$ pelo REAT, $4000 \mathrm{hz}$ por REIL tiveram valores maiores no grupo 2.

Em relação ao protetor auricular do tipo oliva, observou-se também valores maiores no grupo 1. Pelo método REIL, os valores foram nulos no grupo 2.

Quanto se compara a média do REAT entre os sujeitos do grupo 1, obtém-se os resultados apresentados na Figura 1.

Observa-se que o protetor auricular tipo concha apresenta valores maiores em relação aos demais tipos de protetores auriculares; e o protetor auricular tipo oliva apresenta os menores valores.

E pelo método REIL, obtém-se resultados apresentados na Figura 2.

Tabela 1 - Média das REAT e REIL nos dois grupos (grupo 1: trabalhadores e Grupo 2: estudante). $\mathrm{N}=\mathbf{3 0}$

\begin{tabular}{ccc|cc}
\hline \multirow{2}{*}{ Frequência (Hz) } & \multicolumn{2}{c|}{ Grupo 1 } & \multicolumn{2}{c}{ Grupo 2 } \\
& REAT & REIL & REAT & REIL \\
\hline 500 & 10,9 & 10,3 & 9,4 & 2,4 \\
1000 & 14,6 & 14,8 & 15 & 5,5 \\
2000 & 26,1 & 22,9 & 19,4 & 10,3 \\
4000 & 25,6 & 18,6 & 13,8 & 10,3 \\
\hline
\end{tabular}


Tabela 2 - Média do REAT e REIL nas frequências de 500 a $4000 \mathrm{~Hz}$ para os diferentes tipos de protetor auricular, nos grupo 1 e grupo 2

\begin{tabular}{|c|c|c|c|c|c|c|c|c|}
\hline & \multicolumn{4}{|c|}{ REAT } & \multicolumn{4}{|c|}{ REIL } \\
\hline & 500 & 1000 & 2000 & $4000 \mathrm{~Hz}$ & 500 & 1000 & 2000 & $4000 \mathrm{HZ}$ \\
\hline \multicolumn{9}{|l|}{ Concha } \\
\hline Grupo 1 & 21,7 & 26,7 & 38,3 & 35 & 18,3 & 33,3 & 38,3 & 27 \\
\hline Grupo 2 & 12,5 & 32,5 & 27,5 & 15 & 17,5 & 30 & 37,5 & 27,5 \\
\hline \multicolumn{9}{|c|}{$\begin{array}{l}\text { Espuma } \\
\text { descartável }\end{array}$} \\
\hline Grupo 1 & 11 & 17 & 35 & 36 & 18,6 & 22 & 28,2 & 28 \\
\hline Grupo 2 & 10 & 12,5 & 17,5 & 10 & 0 & $-4,5$ & $-6,5$ & 7 \\
\hline \multicolumn{9}{|c|}{$\begin{array}{l}\text { Silicone três } \\
\text { flanges }\end{array}$} \\
\hline Grupo 1 & 9,4 & 14 & 21,1 & 24,2 & 5,5 & 7,2 & 15,1 & 14 \\
\hline Grupo 2 & 12,5 & 10 & 22,5 & 17,5 & -2 & -2 & 15 & 17,5 \\
\hline \multicolumn{9}{|l|}{ Oliva } \\
\hline Grupo 1 & 7 & 6,2 & 19 & 12 & 5,6 & 10,2 & 22,4 & 12,6 \\
\hline Grupo 2 & 2,5 & 5 & 10 & 12,5 & -6 & $-1,5$ & -5 & -11 \\
\hline
\end{tabular}

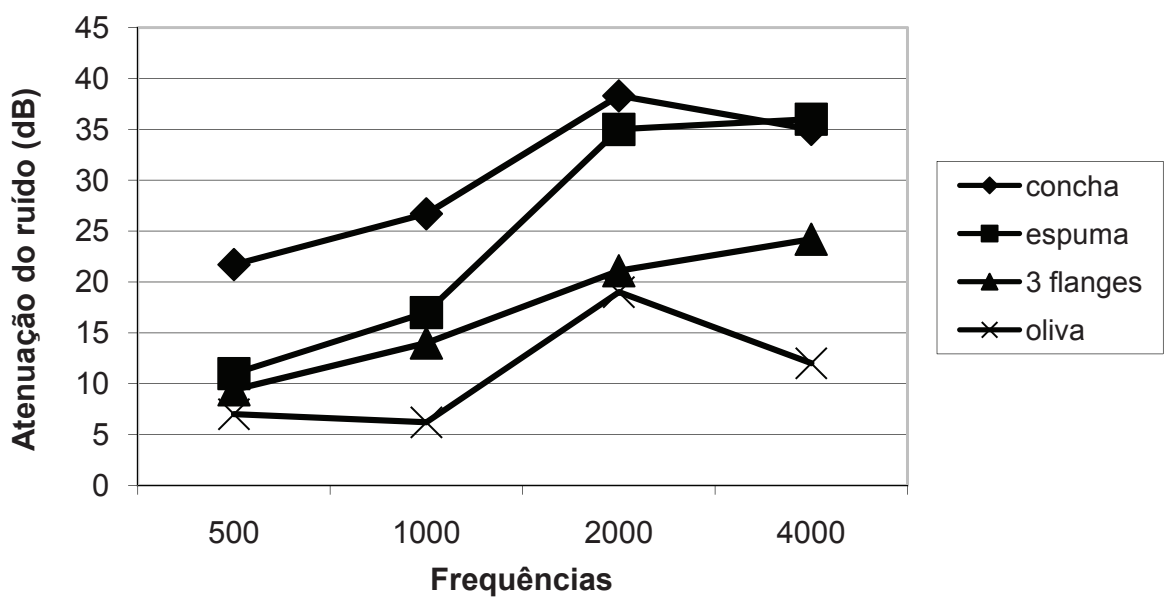

Figura 1 - Média REAT entre 500 a $4000 \mathrm{~Hz}$ nos diferentes protetores auriculares colocados pelos sujeitos do grupo $1(n=23)$

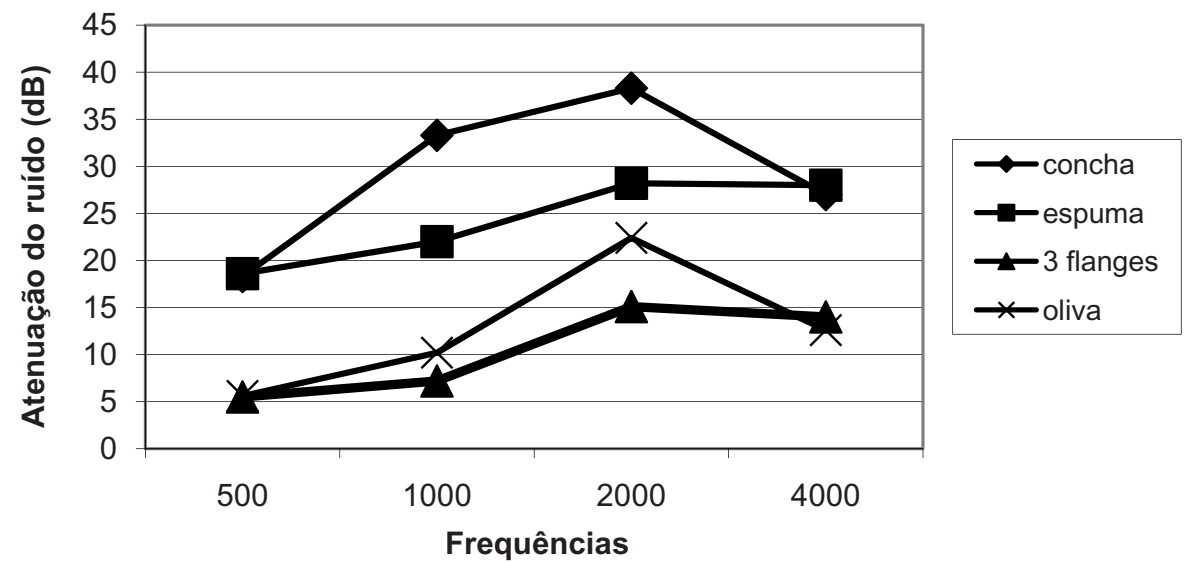

Figura 2 - Média REIL entre 500 e $4000 \mathrm{~Hz}$ nos diferentes protetores auriculares colocados pelos sujeitos do grupo $1(n=8)$ 
Observa-se que o protetor tipo concha e o de espuma descartável também apresentam valores maiores no REIL, mas por esse método, o tipo três flanges obteve menores valores. A frequência de $2000 \mathrm{~Hz}$ apresentou valores maiores que as demais para os quatro modelos de protetores.

\section{DISCUSSÃO}

Nota-se que entre os trabalhadores (grupo 1), treinados e acostumados com a utilização de protetores auriculares, os valores médios de REAT e REIL apresentaram, em geral, valores superiores ao grupo de estudantes (grupo 2) sem treinamento ou hábito de utilização de protetores auriculares (Tabela 1), indicando maior atenuação no primeiro grupo. Conforme referido na literatura, para uma colocação adequada dos protetores auriculares são necessárias orientações precisas e compreensíveis aos trabalhadores, tornando-os hábeis na sua utilização ${ }^{2-4,7}$. Estudo sobre avaliação de protetores auriculares concluíram que quando bem colocados, todos os tipos apresentaram mudanças estatisticamente significantes nos limiares tonais, demonstrando a atenuação dos mesmos ${ }^{18}$. O fato dos estudantes terem um grau de escolaridade maior que os trabalhadores (todos até oito anos de escolaridade), não facilitou a colocação correta do protetor auricular, pode-se dizer que a experiência e treinamento é que contribuem mais significantemente.

Em relação aos métodos utilizados para observar a colocação dos protetores auriculares, na audiometria em campo livre ocorreu certa imprecisão dos limiares de ouvintes normais devido ao mascaramento ambiental causado por ruído da cabina. Além disso, há uma grande variabilidade das medidas em campo livre, sendo relatados por valores de desvio-padrão da audiometria em campo livre de 4 a $6 \mathrm{~dB}^{15}$.

Há uma diferença na frequência de $4000 \mathrm{~Hz}$ (estatisticamente significante no grupo de trabalhadores) que deve ser justificada pela característica da mensuração com microfone-sonda. Pode-se inferir que as atenuações verificadas pelo REIL são menores que as verificadas pelo REAT, devido talvez à perda de inserção ser superestimada. A amplificação natural que ocorre entre as frequências de 2000 a $4000 \mathrm{~Hz}$ é perdida quando o meato acústico externo é ocluído. No entanto, há a criação de um pico de ressonância em frequências mais altas devido ao comprimento residual ser menor. A oclusão do meato provocaria picos de ressonância em torno de $3400 \mathrm{~Hz}{ }^{19}$. Como essa amplificação secundária a oclusão não é considerada pelo método com microfone-sonda, pois este calcula sempre a perda total de ressonância da orelha externa, podendo subestimar a atenuação.

Quando se compara o desempenho da colocação dos protetores auriculares em relação aos diferentes modelos (Tabela 2), observa-se que apesar do tipo espuma descartável ser o que apresenta a melhor atenuação segundo seu fabricante $(29 \mathrm{~dB})$, foi o tipo concha que apresentou maiores valores de REAT e REIL. Isto provavelmente ocorreu por ser esse tipo mais facilmente colocado. Observou-se que para a frequência de $500 \mathrm{~Hz}$ houve valor menor em três dos quatro modelos avaliados, porém essa é uma frequência que não é enfatizada em relação a garantia de melhor atenuação pelos fabricantes de protetores auriculares, que se preocupam em atenuar melhor a banda de frequências altas. Já em outro estudo ${ }^{18}$ foi o tipo espuma descartável que conseguiu um melhor desempenho, com mairo mudança de limiares auditivos avaliados sem e com protetor. As diferenças de atenuação entre os diferentes tipos de protetores auriculares e entre as frequências do ruído são citadas na literatura ${ }^{13}$.

Pela avaliação com os dois métodos, pode-se observar que um melhor desempenho depende do tipo de protetor auricular, mesmo entre sujeitos treinados e acostumados com a utilização de protetores auriculares. Nas Figuras 1 e 2, observa-se que o protetor do tipo concha apresenta valores superiores que os demais tipos. $E$ entre os protetores de inserção, o modelo de espuma descartável, considerado de difícil colocação, superou os demais. Quando se observa o grupo 2, sem treinamento (Tabela 2), o protetor do tipo concha apresenta valores superiores, mas entre os protetores de inserção, é o modelo três flanges o de maiores valores, talvez por ser o de mais fácil colocação entre os três tipos de estudo realizado ${ }^{10}$.

Os métodos utilizados para a avaliação da colocação dos protetores foram eficientes e complementares. Sugere-se outros estudos comparando os resultados dos dois métodos de avaliação de protetores auriculares empregados nesse estudo para validá-los, e um estudo em uma mesma população, porém antes e após treinamento intensivo, comparando o desempenho do grupo.

\section{CONCLUSÃO}

Existem diferenças quanto à colocação de protetores auriculares entre sujeitos treinados e com experiência de uso de protetores auriculares e nãotreinados e sem experiência anterior com protetores auriculares. Nos sujeitos do grupo de trabalhadores (treinados na colocação dos protetores auriculares) a diferença entre os limiares auditivos sem e com protetores, pelos dois métodos utilizados, foram 
superiores aos do grupo 2 (estudantes, sem treinamento), o que evidencia a necessidade de treinamentos para se garantir a correta utilização dos protetores auriculares. Tal fato ficou também evidente quando se observa que o protetor tipo concha, que é de colocação mais simples (apenas ajustá-lo ao redor do pavilhão auricular) teve uma atenuação melhor do que os protetores de inserção, de colocação menos simples.

Há diferenças quando a proteção frente ao ruído de acordo também com o tipo do protetor auricular, sendo que neste estudo, o protetor auricular do tipo concha obteve melhor desempenho e o tipo oliva, o pior.

\begin{abstract}
Purpose: to analyze the effectiveness for inserting different hearing protectors in trained and nontrained subjects. Methods: we investigated the attenuation for four models of hearing protectors (foam earplugs, three-flange silicone earplugs, olive end earplugs and shell earplugs) by means of two strategies, namely, free-field audiometry and microphone probe measurements, with and without h. p. for comparison and determination of noise elimination. Two groups were evaluated: Group 1, with workers trained (23) in the use of hearing protectors, and Group 2, with non-trained ones (8). Results: in group 1, the differences of hearing thresholds with and with no hearing protectors evaluated by the aforementioned methods, in general values, are greater than those of group 2. Shell earplugs submitted average values higher then those of the other types; and olive-end earplugs submitted the minor values. Conclusion: training for using hearing protectors is necessary and must be included in Hearing Conservation Programs (HCP).
\end{abstract}

KEYWORDS: Ear Pprotective Devices; Hearing Loss Noise-Induced; Occupational Health; Hearing

\section{REFERÊNCIAS}

1. Brasil. Portaria No. 25 de 29 de dezembro de 1994. Altera a redação da NR-9 e cria o Programa de Prevenção de Riscos Ambientais orientações para elaboração do mapa de riscos. Diário Oficial da União, 30 de dezembro de 1994.

2. Gonçalves CGO, Iguti AM. Análise de programas de preservação da audição em quatro indústrias metalúrgicas de Piracicaba, São Paulo, Brasil. Cad Saúd Públ. 2006; 22(3):609-18.

3. Rodrigues MAG, Dezan AA, Marchiori LLM. Eficácia da escolha do protetor auricular pequeno, médio e grande em programa de conservação auditiva. Rev CEFAC. 2006; 8(4):543-7.

4. Cavalli RCM, Morata TC, Marques JM. Auditoria dos programas de prevenção de perdas auditivas em Curitiba (PPPA). Rev Bras Otorrinolaringol. 2004; 70(3):368-77.

5. Brasil. Norma Regulamentadora n. 6 Equipamentos de Proteção Individual. Cap V, Título II, CLT. Diário Oficial da União de 15 de fevereiro de 1982.

6. Gonçalves CGO, Vilela RAG, Faccin R, Bolognesi TM, Gaiotto RB. Ambiente de trabalho e a saúde do trabalhador: uma proposta de controle do ruído. Interfacehs. Rev Gestão Integr Saúde Trab Meio Ambiente. 2008; 3(2):2-19.
7. Rabinowitz PM, Duran R. Is acculturation related to use of hearing protection? AlHAJ. 2001; 62(5):611-4.

8. Bockstael A, Keppler H, Dhooge I, D'haenens W, Maes L, Philips B, et al. Effectiveness of hearing protector devices in impulse noise verified with transiently evoked and distortion product otoacoustic emissions. Int J Audiol. 2008; 47(3):119-33.

9. Costa MJ, Menegotto $\mathrm{IH}$, Couto $\mathrm{CM}$, lório MCM. Variabilidade das medidas dos efeitos de ressonância da orelha externa na prática clínica em ambiente não tratado acusticamente. Pró-Fono. 1994; 6(2):14-7.

10. Woxen C, Borchgrevink HM. Attenuation of hearing protector at $85 \mathrm{~dB}$ SPL investigated by commercial insertion gain method. Scandinav Audiol. 1991; (Supl34):145-55.

11. Bramatti L, Morata TC, Marques JM. Ações educativas com enfoque positive em programa de conservação auditiva e sua avaliação. Rev CEFAC. 2008; 10(3):398-408.

12. Toivonen $M$, Paakkonen $R$, Savolainen $S$, Lehtomaki K. Noise attenuation and proper insertion of earplugs into ear canals. Ann Occup Hyg. 2002; 46(6):527-30.

13. Neitzel R, Somers S, Seixas N. Variability of realworld hearing protector attenuation measurements. Ann Occup Hyg. 2006; 50(7):679-91. 
14. Davis RR. What do we know about hearing protector comfort? Noise Health. 2008; 10(40): 83-9.

15. El Dib RP, Attallah AN, Andriolo RB, Soares BGO, Verbeek J. A systematic review of the interventions to promote the wearing of hearing protection. Sao Paulo Med J. 2007; 125(6):359-61.

16. Couto CM, Costa MJ, Almeida K. Mensurações com microfone sonda. In: Almeida K. Iorio MCM. Prótese Auditiva. 2. ed. São Paulo: Lovise; 2003. p.273-304.
17. Katz J. Tratado de audiologia clínica. 3. ed. São Paulo: Manole; 1989.

18. Avagliano A, Almeida K. Estudo do desempenho de diferentes tipos de protetores auriculares. Rev CEFAC. 2001; 3(1):77-87.

19. Couto $C M$. O efeito da ressonância da orelha média e externa na captação das emissões em seres humanos. [tese] São Paulo (SP): Universidade de São Paulo; 2004.

RECEBIDO EM: 22/08/2007

ACEITO EM: 28/05/2008

Endereço para correspondência:

Claudia Giglio de Oliveira Gonçalves

Rua Felisberto Fiore Darozio, 146

Curitiba - PR

CEP: 82410-460

E-mail: clgiglio@terra.com.br 